ARTIGO ORIGINAL

\title{
Pré-penetração e período de latência de Colletotrichum tamarilloi em clones de seringueira resistentes e suscetível sob diferentes condições ambientais
}

\author{
Pre-penetration and latency period of Colletotrichum tamarilloi in \\ resistant and susceptible rubber clones under different environmental \\ conditions
}

\author{
Izabela Ponso Magalhaes ${ }^{1}$ (1D, Marcela Eloi Gomes ${ }^{1}$ (1), Erivaldo José Scaloppi Junior ${ }^{2}$ (ㅇ, \\ Ivan Herman Fischer ${ }^{3}$ (D), Edson Luiz Furtado ${ }^{4}$ (D), Bruno Rafael de Almeida Moreira ${ }^{5}$ (D), \\ Evandro Pereira Prado ${ }^{1}$ (D), Ana Carolina Firmino ${ }^{1}$ (D) \\ ${ }^{1}$ Universidade Estadual Paulista Júlio de Mesquita Filho - UNESP, Dracena, SP, Brasil \\ ${ }^{2}$ Instituto Agronômico - IAC, Votuporanga, SP, Brasil \\ ${ }^{3}$ Agência Paulista de Tecnologia dos Agronegócios - APTA, Bauru, SP, Brasil \\ ${ }^{4}$ Universidade Estadual Paulista Júlio de Mesquita Filho - UNESP, Botucatu, SP, Brasil \\ ${ }^{5}$ Universidade Estadual Paulista Júlio de Mesquita Filho - UNESP, Ilha Solteira, SP, Brasil
}

Como citar: Magalhaes, I. P., Gomes, M. E., Scaloppi, E. Jr., Fischer, I. H., Furtado, E. L., Moreira, B. R. A., Prado, E. P., \& Firmino, A. C. (2021). Pré-penetração e período de latência de Colletotrichum tamarilloi em clones de seringueira resistentes e suscetível sob diferentes condições ambientais. Scientia Forestalis, 49(131), e3630. https://doi.org/10.18671/scifor.v49n131.13

\begin{abstract}
Resumo
A seringueira (Hevea brasiliensis), tem como importância a produção de látex natural que pode ser limitada por vários fatores, dentre eles a ocorrência de doenças, como a antracnose, causada por fungos do gênero Colletotrichum. Os clones IAC 502, IAC 507 e RRIM 937 vêm se mostrando promissores no controle desta doença, pois em alguns estudos vêm apresentando menores porcentagens de antracnose. Assim, o objetivo deste trabalho foi avaliar a pré-penetração e o período de latência de $C$. tamarilloi em clones de seringueira resistentes (IAC 502, IAC 507 e RRIM 937) e suscetível (RRIM 600) sob o efeito de diferentes temperaturas e períodos de molhamento. Para avaliação da germinação e formação de apressórios as temperaturas estudadas foram $15,20,25,30,35$ e $40 \pm 1^{\circ} \mathrm{C}$ e sob períodos de molhamento de $4,6,12$ e 24 horas. O período de latência foi avaliado nas mesmas temperaturas sob períodos de molhamento de 24 horas. A germinação dos esporos e formação de apressórios são diretamente proporcionais ao período de molhamento sendo mais expressivas sob as temperaturas entre 20 a $35^{\circ} \mathrm{C}$ independente do clone. Com 24 horas de molhamento, o clone RRIM 600 mostrou os maiores valores de germinação dos esporos e formação de apressórios. O período de latência foi menor nos clones resistentes e teve influência da temperatura, visto que quanto maior a temperatura menor era o período de latência do fungo na planta. Os resultados apontam para um atraso no desenvolvimento de C. tamarilloi nos clones resistentes, podendo contribuir no atraso do crescimento de taxa de progressão da doença no campo, e diminuindo assim o progresso dela durante o desenvolvimento da cultura.
\end{abstract}

Palavras chaves: antracnose; Hevea brasiliensis; temperatura; molhamento

\section{Abstract}

The rubber tree (Hevea brasiliensis), is an important natural latex-producing plant which can be limited by several factors, such as the occurrence of diseases, like anthracnose, caused by fungi of the genus

Fonte de financiamento: FAPESP (2017 / 23927-3, 2020/11518-4 e 2018/18088-5).

Conflito de interesse: nada a declarar.

Autor correspondente: izabelaponso@gmail.com

Recebido: 13 outubro 2020.

Aceito: 07 janeiro 2021.

Editor: Paulo Henrique Müller Silva. 
Colletotrichum. The clones IAC 502, IAC 507 and RRIM 937 have shown to be promising in the disease control, since in some studies they provide lower percentages of anthracnose. Thus, the objective of this study was to evaluate the pre-penetration and latency period of C. tamarilloi in resistant rubber (IAC 502, IAC 507 and RRIM 937) and susceptible (RRIM 600) clones under the effect of different temperatures and periods of wetting. For germination evaluation and appressorium formation, the studied temperatures were 15, 20, $25,30,35$ and $40 \pm 1^{\circ} \mathrm{C}$ and under wetting periods of $4,6,12$ and 24 hours. The latency period was evaluated at the same temperatures under wetting periods of 24 hours. The percentages of spore germination and appressoria formation were directly proportional to the wetting period, being more expressive under temperatures between 20 to $35^{\circ} \mathrm{C}$, regardless of the clone. After 24 hours of wetting, the clone RRIM 600 showed the highest values of spore germination and appressorium formation. The latency period was shorter in the resistant clones and had an influence on the temperature, since the higher the temperature, the lower the latency period of the fungus in the plant. The results point to a delay in the development of C. tamarilloi in resistant clones, and this can contribute to the delay in the growth rate of disease progression in the field and thus decreasing the amount of disease during a culture phase.

Keywords: anthracnose; Hevea brasiliensis; temperature; wetting

\section{INTRODUÇÃO}

A seringueira (Hevea brasiliensis Müell. Arg.) possui importância econômica devido à produção de látex natural, usado por diversos setores da economia (Alvarenga \& Carmo, 2008). No ano de 2018, o Brasil consumiu cerca de 425 mil toneladas de látex, sendo que mais da metade deste produto foi importado de outros países (Brasil, 2018; IBGE 2018). Entre alguns fatores que podem limitar o aumento na produtividade da cultura no Brasil, estão os problemas fitossanitários. Dentre as doenças que vem acometendo esta cultura encontra-se a antracnose, estando presente em todos os Estados produtores que cultivam seringueira (Furtado \& Trindade, 2005).

Esta doença é causada por fungos do gênero Colletotrichum e afeta diferentes tecidos da seringueira, causando machas cloróticas, seguidas de necróticas que causam a queda prematura da folha e morte apical dos ramos (Silveira et al., 1992). Em alguns casos esta doença também pode ocorrer no painel de sangria, diminuindo a produção de látex pela planta (Furtado \& Trindade, 2005).

Entre os agentes causais da antracnose já relatados no Brasil estão C. gloeosporioides e C. acutatum (Silveira \& Cardoso, 1987; Sierra-Hayer, 2010). Estudos realizados com diferentes isolados de Colletotrichum spp. obtidos de seringais dos estados de Minas Gerais, Bahia, Mato Grosso e Manaus, apontam a possibilidade da existência de outras espécies deste fungo que pertencem a diferentes complexos de espécies (C. acutatum, C. gloeosporioides e C. boninense) (Sarmiento, 2013).Recentemente oito espécies diferentes (C. tamarilloi, C. acutatum, C. scoveillei, C. gloeosporioides, C. siamense, C. fructicola, C. theobromicola e C. karsti) deste patógeno foram identificados em seringueira no Brasil (Antonio, 2020; Gomes et al., 2020). Na Índia e na Colômbia, esta doença foi associada a C.laticiphilum (Damm et al., 2012b), enquanto no Sri Lanka foram identificados C. citri, C. laticiphilum, C. nymphaeae, C. simmondsii e C. acutatum como agentes causadores da antracnose em seringueira (Hunupolagama et al., 2017).

A ocorrência de duas ou mais espécies de Colletotrichum parasitando um mesmo hospedeiro pode dificultar o controle da antracnose, tornando-a uma doença ainda mais importante. Este fato deve-se ao comportamento diferenciado entre espécies, que apresentam sensibilidade diferenciada a fungicidas de diferentes grupos (Tozze Júnior, 2007).

Atualmente o principal método de controle desta doença é baseado na aplicação de fungicidas, principalmente no viveiro (Silveira et al., 1992; Gasparotto et al., 2012). No campo, segundo alguns autores o controle químico em seringueira envolve algumas restrições como: a área foliar deve ser suficiente para permitir a ação eficaz de fungicida; a epidemia não deve estar muito avançada quando o tratamento é iniciado; a intervenção precisa ser muito rápida; muitas vezes é inviável por razões técnicas e econômicas; as copas das árvores devem estar o mais uniforme possível (Guyot et al., 2001). Desta forma, o uso de plantas resistentes seria uma alternativa mais racional e sustentável para controle da antracnose nesta cultura.

A avaliação da reação de 22 clones de seringueira quanto a resistência a antracnose no campo e sob inoculações no laboratório mostram resultados interessantes já que observou-se que no 
campo os clones IAC 507 e IAC 505 foram resistentes a antracnose, pois não apresentaram sintomas da doença no período da avaliação, e em condições de laboratório, seis dias após a inoculação, os clones IAC507, RRIM 937, IAC 505, IAC 502 e IAC 503, apresentaram sintomas de antracnose, porém em menor porcentagem do que os outros clones testados, independente do isolado usado. Vale ressaltar que neste estudo verificou-se que o clone RRIM 600, que é o mais cultivado, se mostrou o mais suscetível à doença, tanto em campo quanto em laboratório (Antonio, 2020).

A reação de resistência de uma planta a um patógeno pode ser definida como a capacidade que ela possui de evitar ou atrasar a entrada ou atividade de um patógeno em seus tecidos, sendo caracterizada por sua natureza dinâmica e coordenada, a qual o sucesso depende da expressão dos mecanismos de resistência da planta em uma sequência lógica, após o contato do patógeno (Pascholati, 2011).Esta interação deve ser vista como um sistema único, que além de depender da planta e do patógeno, também depende do meio ambiente, sendo o único que apresenta alterações frequentes e importantes no decorrer do ciclo de uma cultura, podendo influenciar no grau de suscetibilidade de uma planta e favorecendo ou não a sobrevivência e desenvolvimento do patógeno no hospedeiro ou fora dele (Bedendo \& Amorim, 2011).

No patossitema Colletotrichum-seringueira já existem registros da reação diferenciada de clones à doença de acordo com a região geográfica com microclimas distintos. Por exemplo, o clone GT1 teve maior desfolha ocasionada pela antracnose na região de Matão/SP do que na região de Piracicaba/SP, onde também havia ocorrência da doença (Furtado et al., 1994). Porém, pouco se encontra na literatura sobre o comportamento específico de cada espécie de Colletotrichum em clones de seringueira sob diferentes condições ambientais. Os dados disponíveis na literatura mostram somente o comportamento de $C$. gloeosporioides e C.acutatum, que necessitam de umidade relativa acima de $90 \%$ por 13 horas diárias e temperaturas médias entre 26 e $32^{\circ} \mathrm{C}$ para ocorrência da doença de modo severo (Gasparotto et al., 2012, Wastie, 1972).

A variação no comportamento do clone conforme a região de plantio também pode ser explicada pela variabilidade genética do fungo, que leva a isolados com interação diferenciada com seu hospedeiro, sendo frequentemente relatada no patossitema Colletotrichumseringueira (Saha et al., 2002; Hunupolagama et al., 2017; Antonio, 2020).

Diante do exposto, o presente trabalho teve como objetivo analisar a pré-penetração de C. tamarilloi o período de latência da doença em clones de seringueira que possuem reação de resistência (IAC 502, IAC 207 e RRIM 937) e suscetibilidade (RRIM 600) a antracnose, sob diferentes temperaturas e períodos de molhamento. Com isso podemos ter um conhecimento mais aprofundado sobre a epidemiologia da doença em diferentes condições, colaborando assim no manejo cultural.

\section{MATERIAL E MÉTODOS}

\section{Materiais de seringueiras usadas no trabalho e isolado de C. tamarilloi}

Os folíolos usados no trabalho foram coletados na parte média das plantas dos respectivos materiais genéticos. Os clones IAC 502 (IAC 41 ill.) e IAC 507 (IAC 90 ill.) foram desenvolvidos pelo Instituto Agronômico (IAC) (Gonçalves et al., 2011) e os clones RRIM 600 (Tjir 1 x PB 86) e RRIM 937 (PB 5/51 x RRIM 703) foram desenvolvidos pelo Rubber Research Institute of Malaysia (RRIM) (Wei \& Shamsul Bahri, 2014).

Estes clones apresentam reações de resistência (IAC 507, IAC 502 e RRIM 937) e suscetibilidade (RRIM 600), conforme trabalho desenvolvido por Antonio (2020). As plantas utilizadas para os experimentos encontram-se no jardim clonal do Instituto Agronômico - IAC em Votuporanga, Estado de São Paulo, Brasil. As plantas do jardim clonal têm em média 6 anos idade, e estão plantadas em espaçamento de 1,5m entre linhas e 0,7 metros entre plantas.

Para o desenvolvimento do trabalho foi utilizado um isolado de C. tamarilloi de seringueira armazenado na micoteca de patologia florestal da FCAT/UNESP, caracterizado molecularmente através do sequenciamento de parte das regiões doITS-5.8S rDNA e $\beta$ tubulina (número de acesso no genbank MW031267e MW115955). 


\section{Avaliação da pré-penetração de C. tamarilloi em clones resistentes e suscetível sob diferentes temperaturas e períodos de molhamento}

\section{Inoculação dos folíolos para análise}

O inóculo usado nos experimentos foi obtido por meio do cultivo do isolado em meio de aveia, sob temperatura de $25 \pm 1^{\circ} \mathrm{C}$ e fotoperíodo contínuo, por sete dias. As colônias de cada isolado foram lavadas em água destilada esterilizada e a suspensão obtida filtrada em gaze estéril, quantificada em câmera de Newbauer, e calibrada para $10^{5}$ conídios $\mathrm{mL}^{-1}$.

Folíolos novos destacados da parte apical dos clones RRIM 600, RRIM 937, IAC 502 e IAC 507 de seringueira, previamente desinfetados superficialmente com $\mathrm{NaClO}$ a 2\%, seguida com lavagem com água esterilizada, foram inoculados com alíquotas contendo $30 \mu \mathrm{L}$ de suspensão de conídios $\left(10^{5}\right.$ conídios $\mathrm{m} \mathrm{L}^{-1}$ ) do isolado. A área inoculada foi demarcada com auxílio de adesivos plásticos.

Após a inoculação, os folíolos foram mantidos em câmara úmida a 15, 20, 25, 30, 35 e $40 \pm 1{ }^{\circ} \mathrm{C}$ no escuro. Amostras circulares, com $5 \mathrm{~mm}$ de diâmetro, foram obtidas das áreas inoculadas em intervalos de tempo pré-determinados $(4,6,12$ e 24 horas).

\section{Preparação e avaliação das amostras em microscópio eletrônico de varredura}

Após os intervalos de tempo determinados $(4,6,12$ e 24 horas) as amostras coletadas foram fixadas em solução de "Karnovsky" (glutaraldeido 2,5\%, paraformaldeído 2,0\%, tampão fosfato 0,05M, pH 7,2), por um período mínimo de 2 horas.

As amostras inoculadas foram retiradas do fixador "Karnovsky" e transferidas para microtubos de 1,5 mL contendo tampão cacodilato de sódio $0,05 \mathrm{M}, \mathrm{pH} 7,0$, onde permaneceram por dez minutos, processo repetido por três vezes. As amostras foram pós- fixadas em solução de tetróxido de ósmio $1 \%$ por 2 horas. Subsequentemente as amostras foram imersas por três vezes em água destilada esterilizada e posteriormente desidratadas em série crescente de concentração de acetona (30\%, 50\%, 70\%, 90\% e 100\%) por 10 minutos, sendo o processo repetido por três vezes na concentração de $100 \%$. Após esta etapa, as amostras foram levadas ao aparelho de ponto crítico (Baltec CPD 030) para completar a secagem. Após completa a desidratação, as amostras foram cuidadosamente montadas em "stubs" com fita de carbono dupla face para aderência e cobertas com 20nm de ouro em aparelho Baltec SCD 050. As amostras foram observadas ao microscópio eletrônico de varredura LEO435-VP.

Foram avaliados 100 conídios em Microscópio eletrônico de varredura, em cada repetição, sendo quantificadas as porcentagens de conídios germinados e de apressórios formados. O experimento foi conduzido em delineamento inteiramente casualizado com cinco repetições por tratamento. Os procedimentos formais de Shapiro-Wilk e Bartlett verificaram a normalidade e homogeneidade da variância do conjunto de dados, respectivamente, antes de executar a análise de regressão polinomial

\section{Período de latência de $C$. tamarilloi em clones resistentes e suscetível sob diferentes temperaturas}

Os folíolos dos clones IAC 507, IAC 502, RRIM 937 e RRIM 600 foram levados ao laboratório de Fitopatologia da FCAT-Dracena e inoculados com o isolado de C. tamarilloi.

A inoculação foi realizada através da deposição de uma alíquota de $30 \mu \mathrm{L}$ contendo uma suspensão de conídios $\left(10^{5}\right.$ conídios $\left.\mathrm{mL}^{-1}\right)$ do isolado. A área inoculada foi demarcada com auxílio de adesivos plásticos.

Após a inoculação, os folíolos foram mantidos em câmara úmida (UR de 90\%) sob diferentes temperaturas $\left(15,20,25,30,35\right.$ e $\left.40 \pm 1^{\circ} \mathrm{C}\right)$ sob fotoperíodo alternado de 12 horas. Sabendo que o período de latência de uma doença é o tempo decorrido desde a inoculação até a esporulação (aparecimento dos sinais do patógeno), o experimento foi avaliado diariamente até a visualização a "olho nu" de estruturas do patógeno na área inoculada, sendo que nesse momento o experimento foi dado como finalizado. 
O isolado foi inoculado em cinco folíolos de cada clone, com três repetições por tratamento. Foi utilizado delineamento inteiramente casualizado, sendo que cada câmera úmida continha um tratamento. $\mathrm{O}$ experimento foi realizado duas vezes. Os dados foram submetidos ao Teste de Tukey ao nível de 5\% de probabilidade utilizando o programa SISVAR (Ferreira, 1998).

\section{RESULTADOS E DISCUSSÃO}

A estimativa de germinação dos esporos e formação de apressórios é diretamente proporcional ao período de molhamento, independente do clone avaliado, sendo que as temperaturas de 20 a $35^{\circ} \mathrm{C}$ são as que apresentam maiores valores de germinação (Tabelas 1-5).

Tabela 1. Parâmetros e adequação dos modelos de superfície de resposta para o efeito do período de molhamento e da temperatura na germinação e formação de apressório no clone de seringueira IAC 502

\begin{tabular}{|c|c|c|c|c|c|c|}
\hline \multirow{2}{*}{ Parâmetros } & Estimativa & \multirow{2}{*}{ Erro } & \multirow{2}{*}{ Valor-t } & \multirow{2}{*}{$p>|t|$} & \multirow{2}{*}{$\begin{array}{c}\text { Ponto } \\
\text { estacionário }\end{array}$} & \multirow{2}{*}{$\begin{array}{c}r_{\mathrm{adj}}^{2} \\
0.8920\end{array}$} \\
\hline & Germinação, \% & & & & & \\
\hline$\beta_{0}$, Interceptação & -106.1200 & 5.5230 & -19.2125 & $<2.25 \mathrm{e}^{-16 \star \star \star}$ & & \\
\hline $\begin{array}{l}\beta_{x,} \text { Período de } \\
\text { molhamento, } \mathrm{h}\end{array}$ & 3.7385 & 0.3630 & 10.2985 & $<2.25 \mathrm{e}^{-16 * \star \star}$ & 23.0200 & \\
\hline$\beta_{\mathrm{y}}$, Temperatura, ${ }^{\circ} \mathrm{C}$ & 8.5290 & 0.3855 & 22.1110 & $<2.25 \mathrm{e}^{-16 \star \star \star}$ & 24.7120 & \\
\hline$\beta_{x} \beta_{y}$ & -0.0360 & 0.0065 & -5.6110 & $1.40 \mathrm{e}^{-7 \star \star \star \star}$ & & \\
\hline$\beta_{x}^{2}$ & -0.0620 & 0.0110 & -5.6660 & $1.10 \mathrm{e}^{-7 \star \star \star}$ & & \\
\hline \multirow[t]{2}{*}{$\beta_{y}{ }^{2}$} & -0.1560 & 0.0070 & -22.8370 & $<2.25 \mathrm{e}^{-16 * \star \star}$ & & \\
\hline & Apressório, \% & & & & & 0.1175 \\
\hline$\beta_{0}$, Interceptação & -1.0080 & 0.4165 & -2.4205 & 0.0170 * & & \\
\hline $\begin{array}{l}\beta_{x} \text {, Período de } \\
\text { molhamento, } \mathrm{h}\end{array}$ & 0.0610 & 0.0270 & 2.2405 & 0.0270 * & 19.0300 & \\
\hline$\beta_{y}$, Temperatura, ${ }^{\circ} \mathrm{C}$ & 0.0725 & 0.0290 & 2.5020 & 0.0135 * & 23.4190 & \\
\hline$\beta_{x} \beta_{y}$ & -0.0005 & 0.0005 & -1.0045 & 0.3170 & & \\
\hline$\beta_{x}^{2}$ & -0.0015 & 0.0010 & -1.5930 & 0.1140 & & \\
\hline$\beta_{y}{ }^{2}$ & -0.0015 & 0.0005 & -2.6370 & $0.0095 * *$ & & \\
\hline
\end{tabular}

Significant code: $* \star \star p<0.001 ; * \star p<0.01 ; * p<0.05$.

Tabela 2. Parâmetros e adequação dos modelos de superfície de resposta para o efeito do período de molhamento e da temperatura na germinação e formação de apressório no clone de seringueira IAC 507

\begin{tabular}{|c|c|c|c|c|c|c|}
\hline \multirow{2}{*}{ Parâmetros } & Estimativa & \multirow{2}{*}{ Erro } & \multirow{2}{*}{ Valor-t } & \multirow{2}{*}{$p>|t|$} & \multirow{2}{*}{$\begin{array}{c}\text { Ponto } \\
\text { estacionário }\end{array}$} & \multirow{2}{*}{$\begin{array}{c}r_{\mathrm{adj}}^{2} \\
0.7910\end{array}$} \\
\hline & Germinação, \% & & & & & \\
\hline$\beta_{0}$, Interceptação & -105.9310 & 8.4930 & -12.4725 & $<2.25 \mathrm{e}^{-16 \star \star \star}$ & & \\
\hline $\begin{array}{l}\beta_{x,} \text { Período de } \\
\text { molhamento, } h\end{array}$ & 4.9745 & 0.5580 & 8.9120 & $9.40 \mathrm{e}^{-15 \star \star \star}$ & 22.0740 & \\
\hline$\beta_{y}$, Temperatura, ${ }^{\circ} \mathrm{C}$ & 7.4445 & 0.5930 & 12.5510 & $<2.25 \mathrm{e}^{-16 \star \star \star}$ & 25.2220 & \\
\hline$\beta_{x} \beta_{y}$ & -0.0380 & 0.0100 & -3.8780 & $1.75 \mathrm{e}^{-4 \star \star \star}$ & & \\
\hline$\beta_{x}^{2}$ & -0.0910 & 0.0170 & -5.4075 & $3.55 \mathrm{e}^{-7 \star \star \star}$ & & \\
\hline \multirow[t]{2}{*}{$\beta_{\mathrm{y}}^{2}$} & -0.1310 & 0.0105 & -12.4725 & $<2.25 \mathrm{e}^{-16 \star \star \star}$ & & \\
\hline & Apressório, \% & & & & & 0.3510 \\
\hline$\beta_{0}$, Interceptação & -0.2840 & 0.3365 & -1.1410 & 0.2560 & & \\
\hline $\begin{array}{l}\beta_{x} \text {, Período de } \\
\text { molhamento, } h\end{array}$ & -0.0660 & 0.0220 & -2.9815 & 0.0035 ** & 7.8135 & \\
\hline$\beta_{y}$, Temperatura, ${ }^{\circ} \mathrm{C}$ & 0.0495 & 0.0235 & 2.1085 & $0.0370 *$ & 28.2730 & \\
\hline$\beta_{x} \beta_{y}$ & 0.0015 & 0.0005 & 3.6210 & $0.0005 * \star \star$ & & \\
\hline$\beta_{x}^{2}$ & 0.0015 & 0.0005 & 2.5040 & 0.0135 * & & \\
\hline$\beta_{y}{ }^{2}$ & -0.0010 & 0.0005 & -2.5760 & 0.0110 * & & \\
\hline
\end{tabular}

Significant code: $* * * p<0.001 ; * * p<0.01 ;{ }^{*} p<0.05$ 
Tabela 3. Parâmetros e adequação dos modelos de superfície de resposta para o efeito do período de molhamento e da temperatura na germinação e formação de apressório no clone de seringueira RRIM 600

\begin{tabular}{|c|c|c|c|c|c|c|}
\hline \multirow{2}{*}{ Parâmetros } & Estimativa & \multirow{2}{*}{ Erro } & \multirow{2}{*}{ Valor-t } & \multirow{2}{*}{$p>|t|$} & \multirow{2}{*}{$\begin{array}{c}\text { Ponto } \\
\text { estacionário }\end{array}$} & \multirow{2}{*}{$\begin{array}{c}r_{\text {adj }}^{2} \\
0.9360\end{array}$} \\
\hline & Germinação, \% & & & & & \\
\hline$\beta_{0}$, Interceptação & -129.5900 & 4.7465 & -24.5630 & $<2.20 \mathrm{e}^{-16 \star \star \star}$ & & \\
\hline $\begin{array}{l}\beta_{x} \text { Período de } \\
\text { molhamento, } \mathrm{h}\end{array}$ & 3.8730 & 0.3120 & 12.4160 & $<2.20 \mathrm{e}^{-16 * \star \star}$ & 34.9225 & \\
\hline$\beta_{y}$, Temperatura, ${ }^{\circ} \mathrm{C}$ & 9.1045 & 0.3315 & 27.4655 & $<2.20 \mathrm{e}^{-16 \star \star \star}$ & 22.8290 & \\
\hline$\beta_{x} \beta_{y}$ & -0.0510 & 0.0055 & -9.2640 & $1.45 \mathrm{e}^{-15 \star \star \star}$ & & \\
\hline$\beta_{x}^{2}$ & -0.0390 & 0.0090 & -4.1320 & $6.90 e^{-5 \star \star \star}$ & & \\
\hline \multirow[t]{2}{*}{$\beta_{y}^{2}$} & -0.1605 & 0.0060 & -27.3600 & $<2.20 \mathrm{e}^{-16 \star \star \star}$ & & \\
\hline & Apressório, \% & & & & & 0.5740 \\
\hline$\beta_{0}$, Interceptação & -22.7580 & 3.7080 & -6.0830 & $1.60 \mathrm{e}^{-8 \star \star \star}$ & & \\
\hline $\begin{array}{l}\beta_{x} \text {, Período de } \\
\text { molhamento, } h\end{array}$ & 0.3125 & 0.2435 & 1.2830 & 0.2020 & -11.0540 & \\
\hline$\beta_{y}$, Temperatura, ${ }^{\circ} \mathrm{C}$ & 1.7135 & 0.2590 & 6.6170 & $1.25 \mathrm{e}^{-9 \star \star \star}$ & 28.7880 & \\
\hline$\beta_{x} \beta_{y}$ & -0.0050 & 0.0040 & -1.1535 & 0.2510 & & \\
\hline$\beta_{x}^{2}$ & 0.0075 & 0.0075 & 1.0490 & 0.2965 & & \\
\hline$\beta_{y}^{2}$ & -0.0305 & 0.0045 & -6.7030 & $8.15 \mathrm{e}^{-10 \star \star \star}$ & & \\
\hline
\end{tabular}

Significant code: $* \star * p<0.001 ; * \star p<0.01 ; * p<0.05$

Tabela 4. Parâmetros e adequação dos modelos de superfície de resposta para o efeito do período de molhamento e da temperatura na germinação e formação de apressório no clone de seringueira RRIM 937

\begin{tabular}{|c|c|c|c|c|c|c|}
\hline \multirow{2}{*}{ Parâmetros } & Estimativa & \multirow{2}{*}{ Erro } & \multirow{2}{*}{ Valor-t } & \multirow{2}{*}{$p>|t|$} & \multirow{2}{*}{$\begin{array}{c}\text { Ponto } \\
\text { estacionário }\end{array}$} & \multirow{2}{*}{$\begin{array}{c}\mathrm{radj}^{2} \\
0.7890\end{array}$} \\
\hline & Germinação, \% & & & & & \\
\hline$\beta_{0}$, Interceptação & -90.2995 & 7.7555 & -11.6430 & $<2.20 \mathrm{e}^{-16 \star \star \star}$ & & \\
\hline $\begin{array}{l}\beta_{x} \text { Período de } \\
\text { molhamento, } \mathrm{h}\end{array}$ & 4.1035 & 0.5095 & 8.0510 & $8.75 \mathrm{e}^{-13 \star \star \star}$ & 21.5150 & \\
\hline$\beta_{y}$, Temperatura, ${ }^{\circ} \mathrm{C}$ & 6.9030 & 0.5415 & 12.7450 & $<2.20 \mathrm{e}^{-16 \star \star \star}$ & 24.4440 & \\
\hline$\beta_{x} \beta_{y}$ & -0.0295 & 0.0090 & -3.2875 & $0.0015 * *$ & & \\
\hline$\beta_{x}^{2}$ & -0.0785 & 0.0150 & -5.1210 & $1.25 \mathrm{e}^{-6 \star \star \star}$ & & \\
\hline \multirow[t]{2}{*}{$\beta_{y}^{2}$} & -0.1280 & 0.0095 & -13.3790 & $<2.20 \mathrm{e}^{-16 \star \star \star}$ & & \\
\hline & Apressório, \% & & & & & 0.2140 \\
\hline$\beta_{0}$, Interceptação & -0.8310 & 0.3585 & -2.3175 & 0.0220 * & & \\
\hline $\begin{array}{l}\beta_{x} \text { Período de } \\
\text { molhamento, } \mathrm{h}\end{array}$ & 0.0665 & 0.0235 & 2.8295 & $0.0055 * *$ & 34.1070 & \\
\hline$\beta_{y}$, Temperatura, ${ }^{\circ} \mathrm{C}$ & 0.0515 & 0.0250 & 2.0585 & 0.0420 * & 9.6380 & \\
\hline$\beta_{x} \beta_{y}$ & -0.0010 & 0.0005 & -2.4250 & 0.0170 * & & \\
\hline$\beta_{x}^{2}$ & -0.0010 & 0.0005 & -1.1770 & 0.2415 & & \\
\hline$\beta_{y}^{2}$ & -0.0010 & 0.0005 & -2.0155 & $0.0460 *$ & & \\
\hline
\end{tabular}

Significant code: $* * * p<0.001 ; * * p<0.01 ; * p<0.05$

Tabela 5. Germinação e formação de apressório em clones de seringueira sob ótimas condições ambientais de molhamento e temperaturas para o agente fúngico causador de doenças

\begin{tabular}{|c|c|c|c|c|}
\hline \multirow[b]{2}{*}{ Clone } & \multicolumn{2}{|c|}{ Ponto estacionário } & \multirow{2}{*}{$\begin{array}{l}\text { Ajuste polinomial de } \\
\text { segunda ordem }\end{array}$} & \multirow{2}{*}{$\begin{array}{c}\text { Variável } \\
\text { Germinação, \% }\end{array}$} \\
\hline & $\begin{array}{l}\beta_{x} \text {, Período de } \\
\text { molhamento, } h\end{array}$ & $\beta_{y}$, Temperatura, $^{\circ} \mathrm{C}$ & & \\
\hline IAC 502 & 23.0200 & 24.7100 & $\begin{array}{c}F_{(\alpha, Y)}=-106.1200+3.7380 X+ \\
8.5290 Y-0.0360 X Y-0.0620 \\
X^{2}-0.1560 Y^{2}\end{array}$ & 42.0965 \\
\hline
\end{tabular}


Tabela 5. Continuação...

\begin{tabular}{|c|c|c|c|c|}
\hline \multirow[b]{2}{*}{ Clone } & \multicolumn{2}{|c|}{ Ponto estacionário } & \multirow{2}{*}{$\begin{array}{l}\text { Ajuste polinomial de } \\
\text { segunda ordem }\end{array}$} & \multirow{2}{*}{$\begin{array}{c}\text { Variável } \\
\text { Germinação, \% }\end{array}$} \\
\hline & $\begin{array}{l}\beta_{x}, \text { Período de } \\
\text { molhamento, } h\end{array}$ & $\beta_{y}$, Temperatura, $^{\circ} \mathrm{C}$ & & \\
\hline IAC 507 & 22.0740 & 25.2220 & $\begin{array}{c}F_{(x, Y)}=-105.9310+4.9745 X+ \\
7.4445 Y-0.0380 X Y-0.0910 \\
X^{2}-0.1310 Y^{2}\end{array}$ & 42.8085 \\
\hline RRIM 600 & 34.9225 & 22.8290 & $\begin{array}{c}F_{(x, Y)}=-129.5900+3.8730 X+ \\
9.1045 Y-0.0510 X Y-0.0390 \\
X^{2}-0.1650 Y^{2}\end{array}$ & 39.2960 \\
\hline \multirow[t]{2}{*}{ RRIM 937} & 21.5150 & 24.4440 & $\begin{array}{c}F_{(X, Y)=}-90.2995+4.1035 X+ \\
6.9030 Y-0.0295 X Y-0.0785 \\
X^{2}-0.1280 Y^{2}\end{array}$ & 38.3450 \\
\hline & & & & Apressório, \% \\
\hline IAC 502 & 19.0300 & 23.4190 & $\begin{array}{c}F_{(\alpha, Y)}=-1.0080+0.0610 X+ \\
0.0725 Y-0.0005 X Y-0.0015 \\
X^{2}-0.0015 Y^{2}\end{array}$ & 0.2620 \\
\hline IAC 507 & 7.8135 & 28.2730 & $\begin{array}{c}F_{(X, Y)=}=-0.2840-0.0660 X+ \\
0.0495 Y+0.0015 X Y+0.0015 \\
X^{2}-0.0010 Y^{2}\end{array}$ & 0.2235 \\
\hline RRIM 600 & -11.0540 & 28.7880 & $\begin{array}{c}F(x, Y)=-22.7580+0.3125 X+ \\
1.7135 Y-0.0050 X Y+0.0075 \\
X^{2}-0.0305 Y^{2}\end{array}$ & 0.3465 \\
\hline RRIM 937 & 34.1070 & 9.6380 & $\begin{array}{c}F_{(X, Y)}=-0.8310+0.0665 X+ \\
0.0515 Y-0.0010 X Y-0.0010 \\
X^{2}-0.0010 Y^{2}\end{array}$ & 0.3485 \\
\hline
\end{tabular}

Quando se compara a germinação dos esporos entre os diferentes clones é possível perceber que o clone RRIM 600, mais suscetível à doença, apresenta maiores porcentagens somente quando o período de molhamento é de 24 horas (Figura 1 e 2). Deste modo, os esporos ainda são capazes de germinar em plantas que são resistentes (IAC 507, IAC 502 e RRIM 937) (Figura 3).
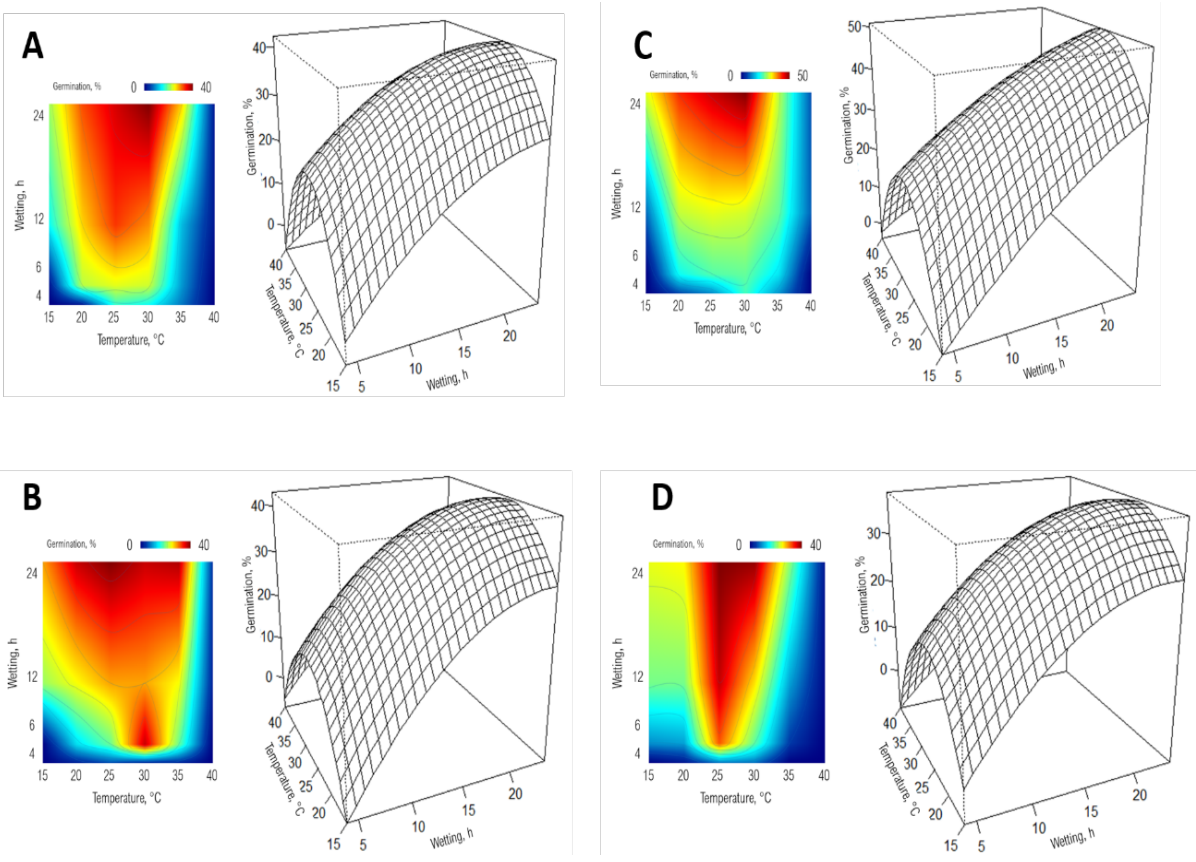

Figura 1: Porcentagem média de esporos germinados nos clones IAC 502, IAC 507, RRIM600 e RRIM937 de $C$. tamarilloi em A, B, C e D respectivamente em diferentes temperaturas $\left({ }^{\circ} \mathrm{C}\right)$ submetidos a diferentes períodos de molhamento. Abordagem de plotagem de contorno difuso e modelo de superfície de resposta de segunda ordem. 

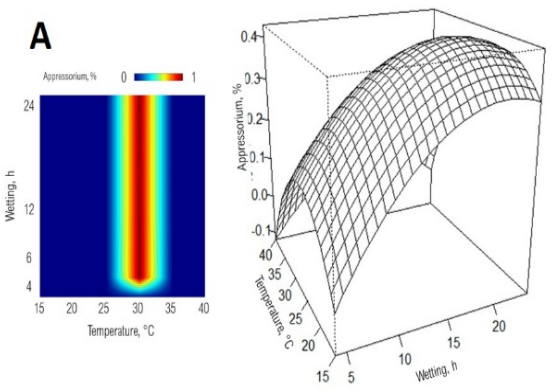

B
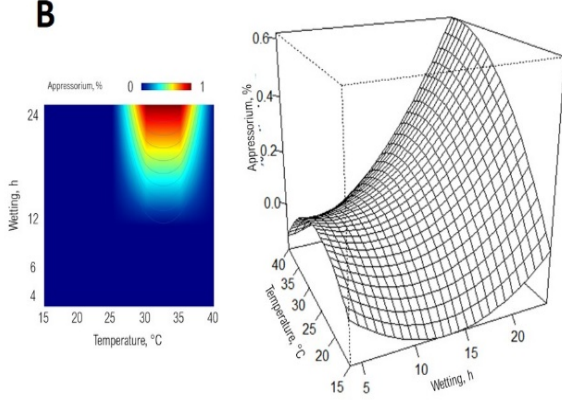
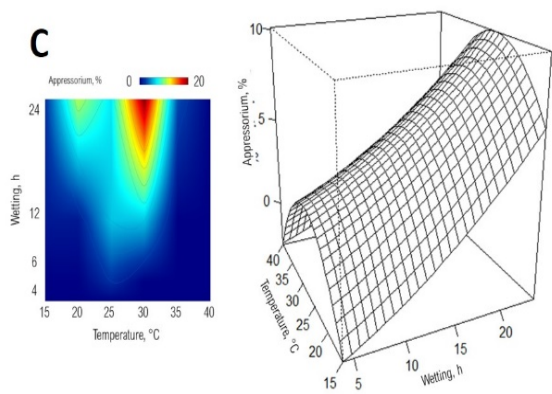

D
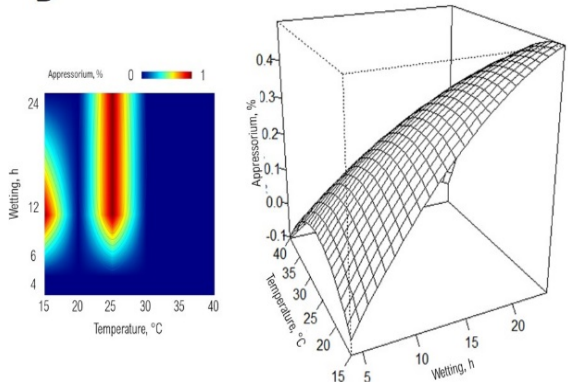

Figura 2: Porcentagem média de apressórios nos clones IAC 502, IAC 507, RRIM600 e RRIM937 de

C. tamarilloi em A, B, C e D respectivamente em diferentes temperaturas $\left({ }^{\circ} \mathrm{C}\right)$ submetidos a diferentes períodos de molhamento. Abordagem de plotagem de contorno difuso e modelo de superfície de resposta de segunda ordem.

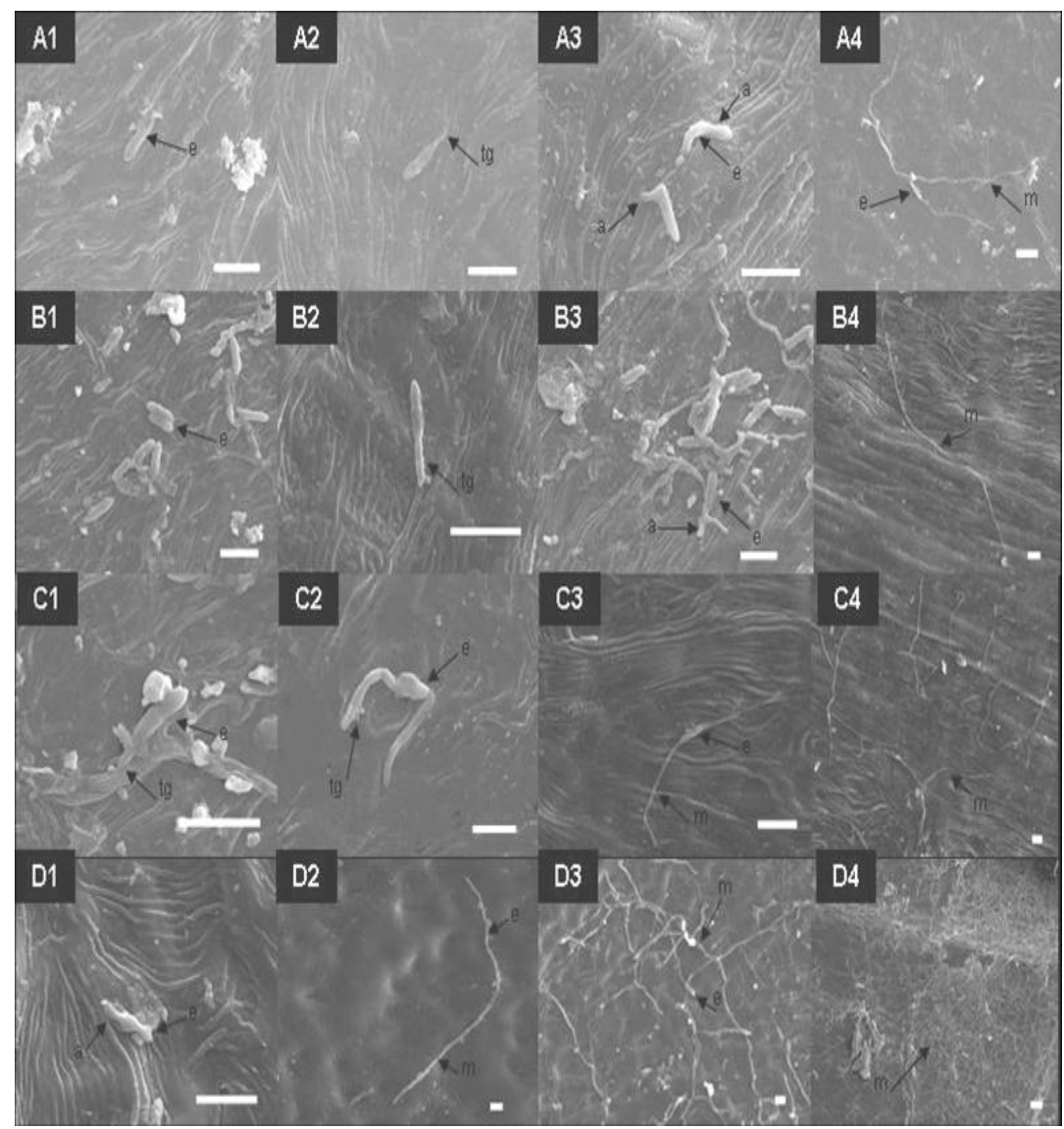

Figura 3: Germinação e formação de apressório a $30^{\circ} \mathrm{C}$ de C. tamarilloi em clones IAC 502 (A), IAC 507 (B), RRRIM 937 (C) e RRRIM 600 (D). 1. 4 horas de molhamento; 2. 6 horas de molhamento; 3.12 horas de molhamento. 4. 24 horas de molhamento. a: apressório; tg: tubo germinativo; m: micélio; e: esporo. Bars: $20 \mu \mathrm{m}$. 
O que difere de maneira significativa entre os clones é formação de apressório, sendo que o clone RRIM 600 apresenta maiores taxas de desenvolvimento desta estrutura independente do período de molhamento, principalmente nas temperaturas de 30 e $35^{\circ} \mathrm{C}$. Notou-se que, apesar de todos os clones exibirem suas superfícies foliares com hifas desenvolvidas sobre a superfície foliar nos períodos de molhamento entre 12 e 24 horas, o clone RRIM600 mostra um desenvolvimento mais avançado, o qual foi possível verificar a fase reprodutiva a $30^{\circ} \mathrm{C}$ com 24 horas de molhamento, sendo que há indícios de que a penetração do fungo ocorra com 12 horas de molhamento nesta mesma temperatura (Figura 3). Assim, nesta etapa do experimento já é possível inferir que o período de latência do fungo no clone suscetível é mais curto do que nos clones resistentes.

Nas inoculações realizadas no laboratório foi observado que o período de latência teve influência da temperatura, sendo que quanto maior a temperatura menor é esse período (Figura 4). Os clones IAC 502, IAC 507 e RRIM 937 apresentam períodos de latência maiores, confirmando as observações realizadas anteriormente em microscópio eletrônico.

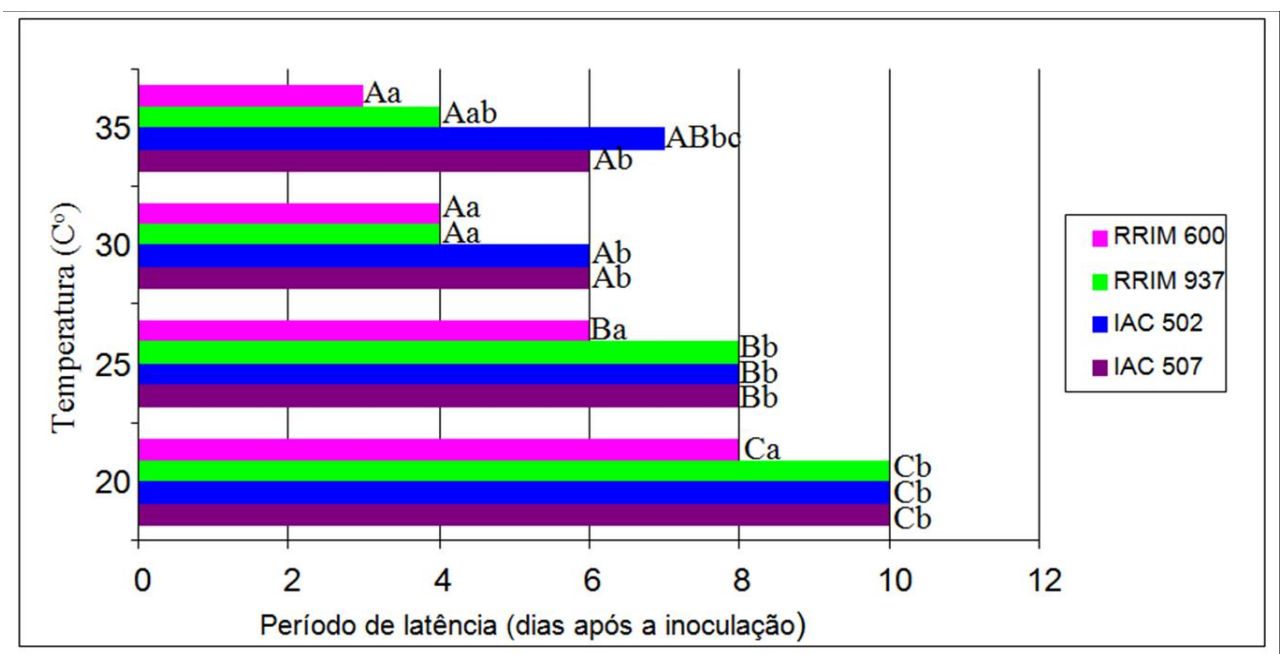

Figura 4: Período de latência de $C$. tamarilloi em diferentes clones de seringueira sob diferentes temperaturas $\left({ }^{\circ} \mathrm{C}\right) .{ }^{*}$ Médias seguidas da mesma letra minúsculas não diferem entre si entre os clones em uma mesma temperatura pelo teste de Tukey a 5\%. Médias seguidas da mesma letra maiúsculas não diferem entre si no mesmo clone em diferentes temperaturas pelo teste de Tukey a 5\%. CV: 15,8\%.

Apesar de se ter avaliado as temperaturas de 15 e $40^{\circ}$, não foi possível coletar nenhum dado, visto que as folhas inoculadas pelo método adotado em laboratório ficam escuras 14 dias após a inoculação, devido a senescência da planta. Assim, o que pode se aferir é que até neste período não foram visualizadas estruturas fúngicas do patógeno inoculado.

As temperaturas entre 25 a $35^{\circ} \mathrm{C}$ se mostraram favoráveis para a germinação e formação de apressório de $C$. tamarilloi em todos os clones estudados. Estes dados corroboram com os resultados obtidos com $C$. gloeosporioides e $C$. acutatum em diferentes materiais de Hevea brasiliensis, pois foram verificadas faixas aproximadas para o desenvolvimento e esporulação, e assim como no nosso trabalho, que a viabilidade e germinação de esporos é diretamente proporcional ao período de molhamento (Wastie,1972).Estes resultados são importantes, pois mostram que apesar de pertencerem a espécies distintas, o comportamento delas são parecidos quando se leva em consideração a temperatura e a umidade.

Diante das informações obtidas sabemos que haverá dificuldade no desenvolvimento de sistemas de previsão de doenças com base somente na variável temperatura (Moraes, 2009). Dessa forma, o que poderia colaborar no manejo dessa doença em campo seria o monitoramento das chuvas, pois o período de molhamento influenciou diretamente no desenvolvimento do patógeno, combinado com o uso de clones que apresentam menores taxas de desenvolvimento da doença. 
A combinação de conhecimentos sobre a influência de fatores ambientas, uso de clones com graus de resistência, combinações de materiais na hora da enxertia da copa e aplicação de produtos químicos, já é uma prática comum na cultura da seringueira para o escape e manejo da doença do mal-das-folhas, causada pelo fungo Microcyclus ulei. Estudos relacionados à influência do ambiente sobre $M$. ulei subsidiaram o zoneamento climático para a implantação da cultura da seringueira no Brasil de modo a escapar das condições ideais para ocorrência do mal-das-folhas, o que fez a região sudeste ganhar destaque na implantação de seringais e produção de borracha natural (Pinheiro et al., 2002; Gasparotto et al., 2012).

Apesar da germinação ser observada em plantas que se mostraram resistentes, é possível observar que há um atraso no desenvolvimento desses esporos quando se compara com o clone RRIM 600, que é suscetível (Figura 1 e 2). No clone suscetível, na temperatura de $30^{\circ} \mathrm{C}$, a partir das 12 horas de molhamento, é possível observar um desenvolvimento expressivo de micélio, apontando uma colonização do hospedeiro mais avançada. Com 24 horas de molhamento foliar é possível visualizar a formação de novos esporos neste clone apontando que este tem um período de latência mais curto, corroborando com os dados obtidos no experimento de período de latência, que mostra uma diferença de dois dias na visualização (em condições de temperatura e umidade ótimas para a germinação e formação de apressório), sem auxílio de microscópio, da reprodução do patógeno.

A diferença na porcentagem de formação de apressório de $C$. tamarilloi entre os clones avaliados pode colaborar na compreensão da resistência e suscetibilidade destes. O apressório é uma estrutura especializada que promove a adesão do patógeno na superfície foliar do hospedeiro facilitando sua penetração. Esta estrutura é formada em resposta a sinais físicos específicos e topografia da superfície da folha (Staples \& Macko, 1980). No caso de Colletotrichum há evidencia que a formação de apressório ainda envolve sinais químicos da planta como ácido clorogênico e fenóis (Harper \& Swinburne, 1979; Parberry \& Blakeman, 1978). Os componentes cuticulares das plantas estão envolvidos na diferenciação da superfície de desenvolvimento do fungo e consequentemente a formação do apressório por ele. Existem evidências da diminuição significativa na formação de apressório em C. gloeosporioides quando colocado para se desenvolver sob a cera contida em plantas não hospedeiras deste patógeno (Podila, Rogers, Kolattukudy, 1993). Assim, estudos mais aprofundados sobre aspectos estruturais e bioquímicos dos clones de seringueira seriam interessantes para podermos explicar esta baixa taxa de desenvolvimento de apressórios sobre a superfície das folhas de clones resistentes.

Quando em condições favoráveis de ambiente e sob a pressão de patógenos agressivos, diferentes indivíduos de uma mesma espécie vegetal podem ser classificados com diferentes níveis de resistência, de acordo com a duração da latência do patógeno. Além do ambiente o período de latência ainda pode sofrer variações de acordo a com o tipo e idade da folha da planta (Amorim \& Pascholati, 2011). Levando em consideração estes pontos, somado aos dados obtidos no presente trabalho, é possível entender a variação de resistência dos clones IAC 502, IAC 507 e RRIM 937 descritos por Antonio (2020), quando avaliados em campo e em laboratório. No campo o clone IAC 507 não demonstrou sintomas de antracnose e os clones IAC 502 e RRIM 937 tiveram baixa quantidade de doença, já em avaliações no laboratório estes mesmos clones apresentaram uma porcentagem de área foliar lesionada superior à do campo. As principais diferenças entre as duas situações (campo e laboratório) são as condições ambientais, que não estavam ideais no campo, e as folhas que foram coletadas ao acaso no campo. Porém, independente da situação, estes clones mostram-se mais resistentes à antracnose do que o clone RRIM 600, pois apresentam menor desenvolvimento de $C$. tamarilloi e maiores períodos de latência. Isso pode contribuir no atraso do crescimento de taxa de progressão da doença no campo, pois sabe-se que quanto mais longo o período de latência maior é a resistência da planta à colonização do patógeno e consequentemente menor ciclo do patógeno será reproduzindo sobre aquele indivíduo e menor será a quantidade de doença no final da cultura (Hau \& Vallavieille-Pope, 1998; Amorim \& Pascholati, 2011). 
Os resultados obtidos no presente trabalho mostram alguns aspectos que estão ligados à resistência de clones de seringueira e abre possibilidades para estudos mais aprofundados sobre o patossistema Colletotrichum-seringueira, colaborando desta maneira no manejo da doença no campo.

\section{CONCLUSÃO}

A germinação dos esporos e formação de apressórios de C. tamarilloi são diretamente proporcionais ao período de molhamento e são mais expressivas sob as temperaturas entre 20 a $35^{\circ} \mathrm{C}$, independente do clone.

A germinação dos esporos de C. tamarilloi somente é maior no clone suscetível, RRIM 600 , no período de molhamento de 24 horas.

A formação de apressórios é mais expressiva no clone RRIM 600 em comparação com os clones resistentes (IAC 502, IAC 507 e RRIM 937).

O período de latência é menor no clone RRIM 600 quando comparado com os clones IAC 502, IAC 507 e RRIM 937, e sofre influência da temperatura.

\section{AGRADECIMENTOS}

Os autores agradecem a FAPESP pelo suporte financeiro e pela bolsa (2017/23927-3, 2020/11518-4 e 2018/18088-5), a APTA e ao Centro de microscopia eletrônica da ESALQ.

\section{REFERÊNCIAS BIBLIOGRÁFICAS}

Alvarenga, A. A., \& Carmo, C. A. F. (2008). Seringueira (894 p.). Viçosa: EPAMIG.

Amorim, L., \& Pascholati, S. F. (2011). Ciclo das relações patógeno-hospedeiro. In: L. Amorim, A. Bergamin Filho \&J. A. M. Rezende. Manual de fitopatologia: princípios de conceitos (4. ed., Vol. 1, pp. 59-98). Piracicaba: Agronômica Ceres.

Antonio, G. L. (2020). Caracterização e identificação molecular, morfo-cultural, fisiológica e patogênica de isolados de Colletotrichum spp. de seringueira e avaliação de resistência de diferentes clones a esse patógeno. Ilha Solteira: FEIS/UNESP. Retrieved in 2020, Maio 20, de https://repositorio.unesp.br/bitstream/handle/11449/191865/antonio_gl_me_ilha_par.pdf?sequenc e=3\&isAllowed=y

Bedendo, I. P., \& Amorim, L. (2011). Ambiente e doença. In: L. Amorim, A. Bergamin Filho \&J. A. M. Rezende. Manual de fitopatologia: princípios de conceitos (4. ed., Vol. 1, pp. 133-147). Piracicaba: Agronômica Ceres.

Damm, U., Cannon, P. F., Woudenberg, J. H. C., Johnston, P. R., Weir, B. S., Tan, Y. P., Shivas, R. G., \& Crous, P. W. (2012 b). The Colletotrichum boninense species complex. Studies in Mycology, 73(1), 1-36. PMid:23136457. http://dx.doi.org/10.3114/sim0002.

Ferreira, D. F. (1998). Sisvar - Sistema de análise de variância para dados balanceados (pp. 1-19). Lavras: UFLA.

Furtado, E. L., \& Trindade, D. R. (2005). Doenças da seringueira. In H. Kimati, L. Amorim, A. Bergamin Filho, L. E. A. Camargo \& J. A. M. Rezende. Manual de fitopatologia: doenças das plantas cultivadas (4. ed., pp. 559-569). Piracicaba: Agronômica Ceres.

Furtado, E. L., Silveira, A. R., Gonçalves, O. S., Costa, J. D., Windel, W., \& Segnini Junior, I. (1994). Avaliação de cultivares de seringueira quanto ao desfolhamento causado por Colletotrichum gloeosporioides Penz., no Estado de São Paulo. Fitopatologia Brasileira, 19, 339.

Gasparotto, L., Ferreira, F. A., Santos, A. F., Pereira, J. C. R., \& Furtado, E. L. (2012). Doenças das Folhas. In L. Gasparotto \&J. C. Rezende Pereira. Doenças da seringueira no Brasil (2. ed. rev. atual, Vol. 3, pp. 35-176). Brasília: Editora Embrapa.

Gonçalves, P. S., Scaloppi Júnior, E. J., Martins, M. A., Moreno, R. M. B., Branco, R. B. F., \& Gonçalves, E. C. P. (2011). Assessment of growth and yield performance of rubber tree clones of the IAC 500 series. Pesquisa Agropecuária Brasileira, 46(12), 1643-1649. http://dx.doi.org/10.1590/S0100204X2011001200009.

Gomes, M. E., Bergamini Lopes, M. P., Fischer, I. H., Tozze Jr, H. J., \& Firmino, A. C. (2020). In: 32 Congresso de Iniciação Cientifica da Unesp, São Paulo. CIC UNESP. 
Guyot, J., Omanda, E. N., Ndoutoume, A., Otsaghe, A. A. M., Enjalric, F., \& Assoumou, H. G. N. (2001). Effect of controlling Colletotrichum leaf fall of rubber tree on epidemic development and rubber production. Crop Protection (Guildford, Surrey), 20(7), 581-590. http://dx.doi.org/10.1016/S02612194(01)00027-8.

Harper, D. B., \& Swinburne, T. R. (1979). 2, 3-Dihydroxybenzoic acid and related compounds as stimulants of germination of conidia of Colletotrichum musae (Berk. \& Curt) Arx. Physiological Plant Pathology, 14(3), 363-370. http://dx.doi.org/10.1016/0048-4059(79)90055-9.

Hau, B., \& Vallavieille-Pope, C. (1998). Wind-dispersed diseases. In D. G. Jones (Ed.), The Epidemiology of Plant Diseases (pp. 323-347). Dordrecht. Kluwer. http://dx.doi.org/10.1007/978-94-017-3302-1_16.

Hunupolagama, D. M., Chandrasekharan, N. V., Wijesundera, W. S. S., Kathriarachchi, H. S., Fernando, T. H. P. S., \& Wijesundera, R. L. C. (2017). Unveiling Members of Colletotrichum acutatum Species Complex Causing Colletotrichum Leaf Disease of Hevea brasiliensis in Sri Lanka. Current Microbiology, 74(6), 747-756. PMid:28378161. http://dx.doi.org/10.1007/s00284-017-1238-6.

Instituto Brasileiro de Geografia e Estatística - IBGE (2018). Recuperado em Setembro de 2020, de https://sidra.ibge.gov.br/tabela/1613

Brasil. Ministério do Desenvolvimento, Indústria e Comércio Exterior. (2018). Recuperado em Setembro de 2020, de http://www.mdic.gov.br/comercio-exterior/estatisticas-de-comercio-exterior/

Moraes, S. R. (2009). Infecção e colonização de Colletotrichum gloeosporioides em goiaba e infecção de Colletotrichum acutatum em folhas de citros 114 p. (Tese de doutorado). Escola Superior de Agricultura "Luiz deQueiroz", Universidade de São Paulo, Piracicaba.

Parberry, D. G., \& Blakeman, J. P. (1978). Effect of substances associated with leaf surfaces on appressorium formation by Colletotrichum acutatum. Transactions of the British Mycological Society, 70(1), 7-19. http://dx.doi.org/10.1016/S0007-1536(78)80164-8.

Pascholati, S. F. (2011). Fisiologia do Parasitismo: como as plantas se defendem dos patógenos. In: L. Amorim, A. Bergamin Filho \&J. A. M. Rezende. Manual de fitopatologia: princípios de conceitos (4. ed., Vol. 1, pp. 593-633) Piracicaba: Agronômica Ceres.

Pinheiro, E., Conceição, H. E. O., Viégas, I. J. M., \& Pinheiro, F. S. V. (2002) Estratégias para controle do maldas-folhas (Microcyclus ulei (H. Henn) V. Arx, na seringueira. Brasília, DF: EMBRAPA. Embrapa Amazônia Oriental-Documentos.

Podila, G. K., Rogers, L. M., \& Kolattukudy, P. E. (1993). Chemical signals from avocado surface wax trigger germination and appressorium formation in Colletotrichum gloeosporioides. Plant Physiology, 103(1), 267-272. PMid:12231933. http://dx.doi.org/10.1104/pp.103.1.267.

Saha, T., Kumar, A., Ravindran, M., Jacob, C. K., Roy, B., \& Nazeer, M. A. (2002). Identification of Colletotrichum acutatum from rubber using random amplified polymorphic DNAs and ribosomal DNA polymorphisms. Mycological Research, 106(2), 215-221. http://dx.doi.org/10.1017/S0953756201005342.

Sarmiento, S. S. (2013). Identificação de espécies de Colletotrichum associadas à antracnose foliar da seringueira (Dissertação de mestrado). Universidade Federal de Viçosa, Viçosa. 72 p.

Sierra-Hayer, J. F. (2010). Caracterização e controle de Colletotrichum spp. em seringueira (Hevea brasiliensis) (Tese de doutorado). Faculdade de Ciências Agronômicas, Universidade Estadual Paulista, Botucatu. $68 \mathrm{p}$.

Silveira, A. P., \& Cardoso, R. M. G. (1987). Ocorrência de Colletotrichum gloeosporioides em seringueira (Hevea brasiliensis) no Estado de São Paulo. Summa Phytopathologica, Piracicaba, 13(1-2), 19.

Silveira, A. P., Furtado, E. L., \& Lopes, M. E. B. M. (1992). Antracnose: nova doença do painel de sangria da seringueira. São Paulo. Instituto Biológico, 18, 196-200.

Staples, R. C., \& Macko, V. (1980). Formation of infection structures as a recognition response in fungi. Experimental Mycology, 4(1), 2-16. http://dx.doi.org/10.1016/0147-5975(80)90045-6.

Tozze Júnior, H. J. (2007). Caracterização e identificação de espécies de Colletotrichum associadas à antracnose do pimentão (Capsicum annuum) no Brasil. (Dissertação de mestrado). Escola Superior de Agricultura "Luiz de Queiroz", Universidade de São Paulo, Piracicaba. 81 p.

Wastie, R. L. (1972). Secondary leaf fall of Hevea brasiliensis: factors affecting the production, germination and viability of spores of Colletotrichum gloeosporioides. Annals of Applied Biology, Cambridge, 72(3), 273-282. http://dx.doi.org/10.1111/j.1744-7348.1972.tb01294.x.

Wei, O. C., \& Shamsul Bahri, A. R. (2014). The introduction of rubber planting recommendations by the rubber research Institute of Malaysia since 1925. Journal of Biology, Agriculture and Healthcare, 4, 49-53. 
Contribuição dos Autores: IPM: Conceituação, investigação, análise formal, curadoria dos dados, validação, escrita; MEG: investigação, curadoria dos dados; EJSJ: investigação, escrita; IHF: investigação, escrita; ELF: investigação, escrita; BRAM: Análise formal e escrita; EPP: Análise formal e escrita; ACF: Conceituação, investigação, análise formal, curadoria dos dados, validação, escrita, administração do projeto. 